\section{AXL TARGETING WITH BEMCENTINB RESTORES PD-1 BLOCKADE SENSITIVITY OF STK11/LKB1 MUTANT NSCLC THROUGH INNATE IMMUNE CELL MEDIATED EXPANSION OF TCF1+ CD8 T CELLS}

${ }^{1}$ Huiyu Li*, ${ }^{2}$ Zhida Liu, ${ }^{1}$ Longchao Liu, ${ }^{1}$ Hongyi Zhang, 'Chuanhui Han, 'Luc Girard, ${ }^{1}$ Hyunsil Park, ${ }^{1}$ Anli Zhang, ${ }^{1}$ Chunbo Dong, ${ }^{1}$ Jianfeng Ye, ${ }^{3}$ Austin Rayford, ${ }^{1}$ Michael Peyton, ${ }^{1}$ Xiaoguang Li, ${ }^{1}$ Kim Avila, ${ }^{1}$ Xuezhi Cao, ${ }^{1}$ Shuiqing Hu, ${ }^{1}$ Esra Akbay, ${ }^{4}$ Luisa Solis, ${ }^{4}$ Carmen Behrens, ${ }^{4}$ Sharia Hernandez-Ruiz, ${ }^{4}$ Lu Wei, ${ }^{4}$ Ignacio Wistuba, ${ }^{4}$ John Heymach, ${ }^{5}$ Michael Chisamore, ${ }^{3}$ David Micklem, ${ }^{3}$ Hani Gabra, ${ }^{3}$ Gro Gausdal, ${ }^{6}$ James Lorens, ${ }^{1}$ Bo Li, ${ }^{1}$ Yang-Xin Fu, 'John Minna, 'Rolf Brekken. 'UT Southwestern Medical Center, Dallas, TX, USA; ${ }^{2}$ SAARI, Taiyuan, China; ${ }^{3}$ BergenBio ASA, Bergen, Norway; ${ }^{4}$ The University of Texas MD Anderson Cancer Center, Houston, TX, USA; ${ }^{5}$ Merck and Co., Inc., Wheaton, IL, USA; ${ }^{6}$ University of Bergen, Bergen, Norway

Background Mutations in tumor suppressor STK11/LKB1 are associated with negative predictive and prognostic impact in NSCLC patients receiving immune checkpoint inhibitors (CPI) in several published cohorts, although there have been some conflicting reports on the association of such mutations with patient outcomes in this setting [1-9]. STK11/LKB1 tumors are characterized by a suppressive tumor micro-environment devoid of cytotoxic T cells, and we hypothesized that targeting the receptor tyrosine kinase AXL, a known driver of an innate immune suppressive microenvironment, would restore sensitivity to PD-1 in syngeneic pre-clinical models as well as in patients harboring STK11/LKB1 mutated NSCLC.

Methods Stk11/Lkb1 (L) mutation was introduced by CRISPR technology into murine lung adenocarcinomas driven by mutant Kras and Trp53 loss (KP). Sensitivity towards anti-PD1 was evaluated in the absence and presence of the small molecule AXL inhibitor bemcentinib in the KPL model and in a human NSCLC xenograft model carrying a STK11/LKB1 mutation. The immune tumor landscape was mapped following introduction of the Stk11/Lkb1 mutation and therapeutic intervention with anti-PD-1/pembrolizumab and bemcentinib. FFPE fine-needle aspirate biopsies of target lesions were acquired from patients at screening immediately prior to enrollment in BGBC008, a PhII single-arm, 2-stage study with bemcentinib (200mg/d) and pembrolizumab (200 mg/q3wk) for previously-treated stage IV lung adenocarcinoma patients who were CPI naïve or CPI refractory. Patients were assessed for response according to RECIST1.1 criteria at scheduled scan intervals.

Results Introduction of a STK11/LKB1 mutation into murine lung adenocarcinomas driven by mutant Kras and Trp53 loss resulted in a PD-1 refractory syngeneic KPL tumor. Mechanistically this occurred because KPL mutant NSCLCs lacked TCF1-expressing CD8 T cells, a phenotype that was recapitulated in human STK11/LKB1 mutant NSCLCs. Systemic inhibition of AXL with bemcentinib resulted in increased type I interferon secretion from dendritic cells resulting in expansion of tumor-associated TCF1+PD-1+CD8 T cells and restored therapeutic response to PD-1. This effect was observed in a syngeneic immunocompetent mouse model and in humanized mice bearing STK11/LKB1 mutant NSCLC human tumor xenografts.In an ongoing clinical trial (NCT03184571), 3 evaluable NSCLC patients with identified STK11/LKB1 mutations demonstrated objective clinical response/clinical benefit to the combination of AXL inhibitor bemcentinib and pembrolizumab Conclusions In these models, AXL is a critical targetable driver of immune suppression in STK11/LKB1 mutant NSCLC contributing to CPI resistance. Our results show that inhibition of AXL rescues this deficit and represents a new clinical strategy in combination with anti-PD-1 therapy in NSCLC patients carrying a STK11/LKB1 mutation

Acknowledgements The authors would like to thank all patients and their caretakers for participating in this trial.

Trial Registration Patients treated with bemcentinib and pembrolizumab combination therapy were enrolled in the BGBC008 clinical trial (BerGenBio ASA and Merck \& Co., Inc., Kenilworth NJ, USA, NCT03184571)

\section{REFERENCES}

1. Gu M, Xu T, Chang P. KRAS/LKB1 and KRAS/TP53 co-mutations create divergent immune signatures in lung adenocarcinomas. Ther Adv Med Oncol. 2021:13:17588359211006950

2. Cho BC, Lopes G, Kowalski DM. Relationship between STK11 and KEAP1 mutational status and efficacy in KEYNOTE-042: pembrolizumab monotherapy as firstline therapy for PD-L1 positive advanced NSCLC. Cancer Res. 2020;80(16 Supplement): CT084.

3. Aredo JV, Padda SK, Kunder CA. Impact of KRAS mutation subtype and concurrent pathogenic mutations on non-small cell lung cancer outcomes. Lung Cancer. 2019;133:144-150

4. Kwack WG, Shin SY, Lee SH. Primary Resistance to Immune Checkpoint Blockade in an STK11/TP53/KRAS-Mutant Lung Adenocarcinoma with High PD-L1 Expres sion. Oncol Targets Ther. 2020:13:8901-8905.

5. Shire NJ, Klein AB, Golozar A. STK11 (LKB1) mutations in metastatic NSCLC Prognostic value in the real world. PLoS One. 2020;15(9):e0238358. 6. Skoulidis F, Goldberg ME, Greenawalt DM. STK11/LKB1 Mutations and PD-1 Inhibitor Resistance in KRAS-Mutant Lung Adenocarcinoma. Cancer Discov. 2018;8 (7):822-835. 7. Wang H, Guo J, Shang X. Less immune cell infiltration and worse prognosis after immunotherapy for patients with lung adenocarcinoma who harbored STK11 mutation. Int. Immunopharmacol. 2020:84:106574. 8. Kitajima S, Ivanova E, Gou S. Suppression of STING Associated with LKB1 Loss in KRAS Driven Lung Cancer. Cancer Discov. 2019:9(1):34-45. 9. Mograbi B, Heeke S Hofman P. The Importance of STK11/LKB1 Assessment in Non-Small Cell Lung Carcinomas. Diagnostics (Basel). 2021;11(2):196.

Ethics Approval This study was approved by the following ethical committees: Use of human cord blood: UT Southwestern (UTSW) Parkland Hospital, STU 112010-047Animal studies: UTSW Medical Center, Institutional Animal Care and Use Committee, APN 2015-100921Clinical study: London Bridge Research Ethics Committee (UK): 17/LO/0418; REC-South East (Norway): 2017/473; Drug Research Ethics Committee of the University Hospital Clinic of Barcelona (Spain): BGBC008/ MK-3475_PN-531; Medical College of Wisconsin Institutional Review Board \#4 (USA): PRO00029453

http://dx.doi.org/10.1136/jitc-2021-SITC2021.602 